\title{
A Novel Image Retrieval Technique based on Gabor Function, Local Tetra Pattern and ASMC
}

\author{
Kiran Ashok Bhandari \\ Associate Professor: Department of CMPN \\ TCET, Kandivali (E) \\ Mumbai, India
}

\author{
Ramachandra Manthalkar, PhD \\ Professor, E\&TC Dept \\ SGGSIE\&T, Nanded
}

\begin{abstract}
CBIR alone won't give perfect retrieval results due to semantic gap. To overcome the problem of semantic gap in CBIR, more than one Semantic Content Based Image Retrieval techniques are required which is known as Hybrid Classification System. Hence the proposed approach uses multiple machine learning techniques with combination of different classifiers like supervised and unsupervised, soft classifiers, spectral contextual classifiers. Remotely Sensed Image Retrieval System (RSIR) has to identify and retrieve similar images based on query image, to do so we need to extract feature of image in order to compare query Image and database image. The proposed approach is a combination of two Phases. First Phase involves feature extraction by Texture Feature with the help of Gabor Function and Spectral Distribution using Advanced Split and Merge Clustering whereas second Phase identifies the Local Pattern of retrieved images in Phase-I.
\end{abstract}

The performance of the proposed approach is measured in terms of Precision, Recall and f-measure. Statistical analysis of the proposed hybrid approach in Phase-I (Texture and Spectral Distribution) shows that precision, recall and f-measure is get improved, on an average by $19.46 \%, 8.84 \%, 14.46 \%$ respectively when get compared with CBIR (Texture). Phase-I and Phase -II comparison in term of f-measure is increased up to $96.95 \%$. Hence the hybrid approach gives more accurate result as compare to individual approach

\section{General Terms}

Image Retrieval for dataset of satellite imagery

\section{Keywords}

Local Tetra Pattern, Split and Merge Clustering, Gabour Function

\section{INTRODUCTION}

Remote sensing has turned out to be one of the major research applications of image processing domain. Satellites cover different areas like water bodies $\mathrm{m}$ forests, urban and many more which help us in disaster forecasting, monitoring of environment ,geological survey etc. With the continuously expanding demand for RSIR, many satellites have been launched, and thousands of images are acquired every day. This leads to steadily increasing database of these remotely sensed images which makes it difficult for extraction of important information from them. So the retrieval of meaningful images from unstructured database is a difficult and challenging. One of the main purposes of satellite remote sensing is to interpret the observed data and classify features. To obtain high quantitative accuracy we can use all the available brightness levels in satellite images using classification.

Due to rapid growth in digital libraries necessity for huge database has raised. Hence there must be the efficient way to retrieve the images from huge database based on image content. Current method is Image collection exploration which is based on the use of novel exploration paradigms call Image Collection Exploration [19].Image collection exploration is a mechanism to explore huge digital image repositories which can be produced through different devices such as mobile, camera, satellites etc. As CBIR suffers of known problem call semantic gap between the low level image features and high level semantics which can be overcome by Image collection exploration which consists of a set of computational methods to summarize represent, visualize and navigate image repositories in an efficient manner. This can be done by semantic content Based Image Retrieval. There are various techniques that are developed in reducing the semantic gap like Machine Learning Techniques. Supervised and unsupervised, SVM with additional techniques like SVM-BDT [9], Neural Network machine learning techniques are available. Among two broads of classification procedures: supervised classification unsupervised classification. The supervised classification is the essential tool used for extracting quantitative information from remotely sensed image data.

Here the query-by-example approach adopted also to represent the stored senses in an expressive and efficient way, undertook substantial work to extract meaningful features out of satellite images and to develop compact descriptors. Hence text based retrieval fails then to overcome it CBIR comes into picture. CBIR is also known as Visual Image Retrieval, which uses the contents of images to represent and access the image. Content nothing but unique descriptors like shape, color, and texture of images as they are easily taken from the image without the need of any external knowledge base.

\subsection{Objective}

To retrieve remotely sensed images, proposed work designed with following objectives,

1.1.1 To have desired accuracy (more than 90\%) with minimum response time using improves split and merge technique.

1.1.2 To improve performance in terms of precision, recall and f-measure

As abstract nature of extracted data inhibits the usage of similarity measures that were derived from human perception and most similarity measured aren't suitable for content vectors with variable length. Another problem in consent based retrieval is that often the query image does not ramble the search characteristic to the desired accuracy. Hence the main objective is retrieval accuracy with less number of iteration. The performance of method is measured in terms of average precision $(\mathrm{P})$ and average recall $(\mathrm{R})$, collectively f-measure. $\mathrm{P}$ and $\mathrm{R}$ are defining as follow 
$>\quad P=\frac{\text { Number of relevant images retrieved }}{\text { Total Number of images retrieved }}$

$>\quad R=\frac{\text { Total numberofrelevant images in database }}{\text { Number of relevant images retrieved }}$

$>f-$ measure $=\frac{2 R P}{R+P}$

\section{LITERATURE REVIEW}

2.1 T. Bretschneider, R. Cavet, O. Kao "Retrieval of remotely sensed imagery using spectral information content" [1], proposed the CBIR using simple unsupervised query-byexample approach that can have exploitation of spatial features i.e. not only characterized by shape but by texture also (special distribution of spectral information) which will help to increase retrieval accuracy but just one feature exploitation

2.2 T.S. Lee "Image representation using 2D Gabor wavelets" [5], proposed the extension to two dimensions frame criterion developed by Daubechies for one dimensions wavelets, and it computes the frame bounds for particular case of 2D Gabor wavelets. Earlier work on Gabor wavelets target on issues in image compression and compact representation but here new more relevant issue in brain modeling i.e. precision in resolution achieved through redundancy is get handled.

2.3 Y. Rui, T. S. Huang, S. Mehrotra, "Content-based image retrieval with relevance feedback in MARS" [6],Proposed combination of Image Processing(IP) and information Retrieval (IR) to retrieve image, which will help to increase precision but not average Recall(R).Z. Guo, L. Zhang, and D. Zhang, "A completed modeling of local binary pattern operator for texture classification"[10],address difficulties in weighted local gray level(WLG) to replace the traditional gray value of centre pixel, which was not significant and one more parameter has to be tuned, so with the help of first order derivative and second order derivatives here proposed new approach to increases accuracy also introduced simple and effective strategy, Average Local Gray Level(ALG) which is less sensitive to illumination variations and viewpoint variations.

2.4 En cherg, Feng Jing, Chao, Lei Zhang, "search Result clustering based relevance feedback for web image retrieval" [14], as no commercial web image search engines support relevance feedback(RF) due to issues like salability, efficiency, usability. For example online searching need quick response so to wake in real time environment RF must be fast. In this paper proposed a

Search result clustering (SRC) based RF mechanism for retrieval of web image [15]. To evaluate it automatically an image subset was selected and manually labeled. In experimental results average precision of traditional RF and SRC based RF is 0.5481 and 0.6478 res. It shows that SRC results are good as it remove unimportant and noisy words. Even SRC based RF average time cost is 0.99 which is less than traditional RF and only SRC gives average time cost 0.664 .Due to this new proposed technology we could incorporate RF into web image retrieval in practical way. Testing was done on millions of data so it's effective and scalable. But this proposed work covers explicit feedback, didn't consider implicit relevance feedback. Even only textual feature are used in this algorithm hence only that kind of data can be treated, audio and video data without textual feature won't get processed by this method.

\section{METHODOLOGY}

\subsection{Scope of Project}

The complete architecture of the proposed system is shown below in the figure 1.As shown in figure 1 Input query image taken and undergoes pre-processing i.e. resizing and rgb to gray scale conversion of the input image for easy computations, then feature extraction is done to retrieve relevant images from previously stored database as there pattern is already store in database to get it compare with query image [12] [13] that's why this process is known as supervised. Feature extraction and similarity measurement by following methods.

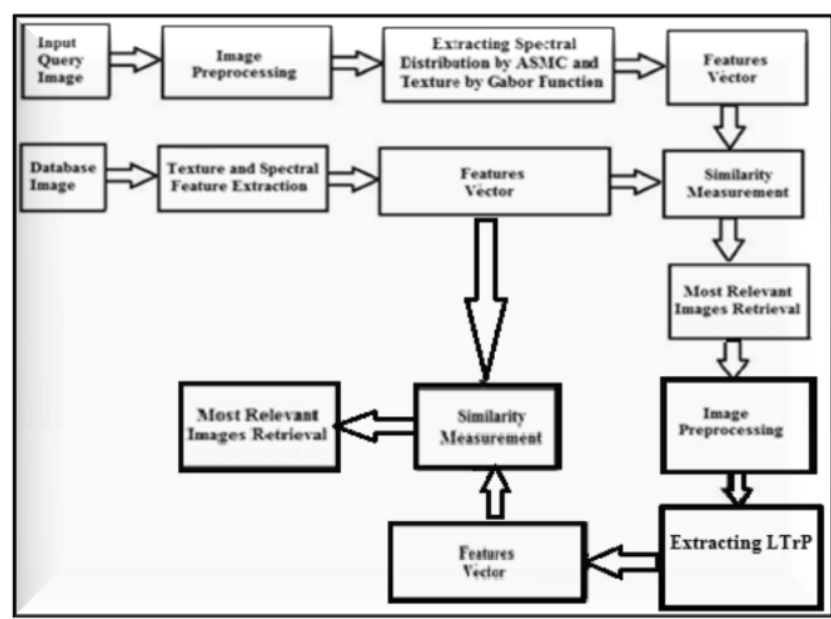

Fig.1. Block Diagram of the Proposed Framework

Phase I:-Supervised Retrieval using piecewise defined pattern similarities

\section{Feature Extraction}

Here two feature are extracted they are

a) Spectral Distribution(SD)

b) Texture Feature (TF)

Pattern form here is concatenation of spectral distribution and texture feature as shown in equation (3). Feature Spectral Distribution implemented by modified parallel version of the Advanced split and merge clustering (ASMC) algorithm [2] and described by the vector $\left\{\left(\mathrm{C}_{1}, \mathrm{~W}_{1}\right), \ldots \ldots,(\mathrm{Cn}, \mathrm{Wn})\right\}$, where $\mathrm{C}_{\mathrm{i}}$ and $\mathrm{W}_{\mathrm{i}}$ are the multidimensional cluster centers and scalar weights of the $\mathrm{i}^{\text {th }}$ cluster, respectively.

$>$ Parallel computation done over here for multiple images is in form of master and slave:-

\section{Work by master}

1. Initialise process and assign o slaves Till cover all images

2. Send new cluster information to all slaves

3. Split cluster

4. Apply K-mean

5. Merge cluster

Receive results and compute new cluster centres Send cluster information to all slaves

Work by slaves

1. Receive cluster centers from the master 
2. Set all elements to closed cluster centre

3. Compute new cluster centre

4. Give new cluster centre information to master

5. Weight for master information for further processing

> Advanced parallelizes split and merge clustering Algorithm given as follow:

Step 1: One Query image taken (Image I), get pre-processed means convert Image from RGB Color Space to L*a*b* Color Space as shown in fig.4 (i) and fig.4 (ii) and image get divided into $4 * 4$ i.e. 16 blocks by combining row wise as well as columns wise as shown in fig.4(iii) and equation (1). In matlab matrix form get converted into cell.

$$
b l k R=\frac{\text { rows }}{4}, \text { Cblkc }=\frac{\text { columns }}{4}
$$

As ASMC algorithm is parallelized, each block get send by master for clustering to respective slave so that parallel processing done through three slaves based on RGB.

Each slave then Calculate two parameters:-

$$
\text { Scalar Weight }(W)=\frac{1}{W^{\wedge\left(\frac{1}{\text { rollof Factor })}\right.}}
$$

As shown in equation (1) scalar weight of cluster get calculated where 1 is the lowest value in $\mathrm{W}$ at the output is initialized.

\section{While different parameters used are}

$>$ 'RolloffFactor'- Positive scalar that controls how fast the output weight falls as the function of absolute difference between an intensity and the reference grayscale intensity. High value of this parameter means that the output weight values will fall off sharply for intensities that are very different from reference grayscale intensity, whereas low value of this parameter would allow a more gradual falloff. Value in the range [0.5 4] typically found to be useful for this parameter. So the Default value is 0.5 . Where $\mathrm{W}$ is Absolute value and complex magnitude

Calculation of weights for image pixels based on greyscale intensity difference. Computation based on the absolute value of the difference between the greyscale intensity of the pixel and the reference grey scale intensity specified by, which is a scalar. The weights are returned in the array $\mathrm{W}$, which is the same size as input image I. The output weight of a pixel is inversely related to the absolute value of the greyscale intensity difference at the pixel location, so that for pixels with small difference (regions with intensity close to referred Greyscale, output weight is large, and for pixels with large difference (regions with intensity very different from referred Greyscale), output weight is small.

> 'Grayscale Difference Cutoff' - Non-negative scalar, K. This parameter puts a hard threshold on the absolute grayscale intensity difference values, and strongly suppresses the output weight value for any pixel whose absolute grayscale intensity difference from the reference grayscale intensity is greater than $\mathrm{K}$ by assigning them the smallest weight value

Cluster Centers

Calculating gradient in $\mathrm{x}$-direction as well as in $\mathrm{Y}$-direction.
Then combine both of them to get one cluster centre. Each block gets processed on the bases of RGB by individual slave which is get assign by master. This is done with the help of max value in image matrix while process start with iterate from first corner to last corner.

\section{Step 2: Apply K-means}

Classify the Colors in ' $a * b^{* '}$ Space Using K-Means Clustering. Since the color information exists in the ' $a * b^{*}$ ' space, your objects are pixels with ' $a *$ ' and ' $b$ *' values. Use $\mathrm{k}$-means to cluster the objects into three clusters using the Euclidean distance metric.

Step 3: Removals of empty clusters need not to done as it get merge automatically in matlab.

Step 4: Merging of clusters with the help of cluster centres and scalar weights of respective block and then whole merging of clusters. Merging means cell get converted into original image matrix.

Texture characteristic extracted using two-dimensional Gabor functions [12] [3] [4], which analyse an image in the spatialfrequency domain and proved to be a visually effective method [5]. To accommodate for rotational invariance, the Gabor filter wad computed for eight different orientations, after the filtering, the orientation depended coefficients of each image pixel form a vector in the eight dimensional spaces. Finally, most significant textures which group clusters in the vector space were located using the k-means algorism. At last content image of an image is formed by the concatenation of the spectral and the texture descriptor and called as pattern.

$>$ Similarity Measurement

Modified version of the Earth Mover's Distance (EMD) for comparison of the underlying patterns with variable lengths. As not every sub space of one vector can be mapped onto any arbitrary section of another vector EMD modified to be restricted to piecewise predefine parts of each vector. As shown in Fig.2 Transformation cost minimisation which follow equation (3) to (6). Here due to modified EMD each and every sub-space of one vector can be mapped onto an arbitrary section of any other vector.

$\operatorname{Let} P_{Q}=\left\{\left(C_{1}, W_{1}\right), \ldots,\left(C_{n}, W_{n}\right),\left(G_{1}, V_{1}\right), \ldots,\left(G_{m}, V_{m}\right)\right\}$

Be the pattern of the query image. This can be rewritten with consecutively indexes as

$$
P_{Q}=\left\{\begin{array}{c}
\left(C_{1}, W_{1}\right), \ldots,\left(C_{n}, W_{n}\right),\left(G_{n+1}, V_{n+1}\right), \\
\ldots,\left(G_{n+m}, V_{n+m}\right)
\end{array}\right\}
$$

and compare with an arbitrary pattern in the database e.g.

$P_{D B}=\left\{\left(K_{1}, L_{1}\right), \ldots,\left(K_{l}, L_{l}\right),\left(F_{l+1}, Q_{l+1}\right), \ldots,\left(F_{l+k}, Q_{l+k}\right)\right\}$ 


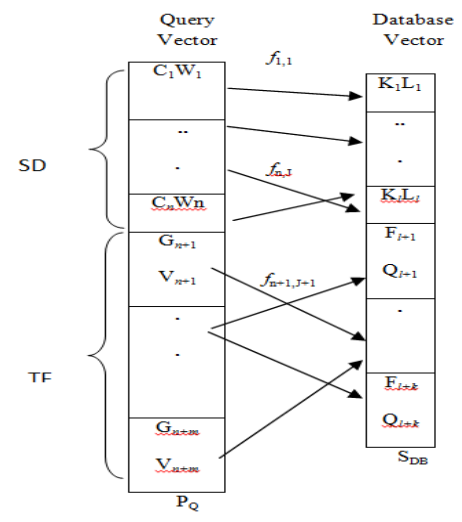

Fig.2.Transformation cost minimization

Since both PQ and PDB consists of a spectral and a textural descriptor, the EMD has to be calculated individually within the two part.[11].The final similarity is defined as

$\operatorname{EMD}\left(\mathrm{P}_{Q}, \mathrm{PDB}\right)=\mathrm{w}_{\mathrm{C}} \mathrm{EMD}_{\mathrm{C}}\left(\mathrm{P}_{Q}, \mathrm{PDB}\right)+\mathrm{w}_{\mathrm{G}} \mathrm{EMD}_{\mathrm{G}}\left(\mathrm{P}_{Q}, \mathrm{P}_{D B}\right)$

Where $\mathrm{w}_{\mathrm{C}}$ and $\mathrm{w}_{\mathrm{G}}$ are heuristically determined weights for the distance based on the spectral content and the textural content.

Finally relevance feedback approach [6] [7] for interactive search strategy using EMD flow matrix [8] to obtain centre correspondences between the patterns. Once we get relevant image from ASMC and Gabor function we apply relevance feedback using LTrP given in section: 4.2 to filter out some more images and get exact similar images from database

\section{Phase II: - Local Tetra Pattern ( LTrP)}

The idea of LTrP is depends on local patterns described in LBP, LTP \& LDP. The LTrP [16] using the direction of the center gray pixel gives the spatial structure of the local texture. Consider an image $\mathrm{I}$, the first-order derivatives along $0^{\theta}$ and $90^{\theta}$ directions are denoted as

$I_{\propto}^{1}\left(g_{p}\right) \mid \alpha=0^{\theta}, 90^{\theta}$

$\mathrm{I}_{0^{\theta}}^{1}=\mathrm{I}\left(\mathrm{g}_{\mathrm{h}}\right)-\mathrm{I}\left(\mathrm{g}_{\mathrm{c}}\right)$

$\mathrm{I}_{90^{\theta}}^{1}=\mathrm{I}\left(\mathrm{g}_{\mathrm{v}}\right)-\mathrm{I}\left(\mathrm{g}_{\mathrm{c}}\right)$

Different direction of $\mathrm{g}_{\mathrm{c}}$ formulated as:

$I_{\text {dir }}^{\prime}\left(g_{c}\right)=\left\{\begin{array}{c}1, \quad I_{0^{\theta}}^{1}\left(g_{c}\right) \geq 0 \text { and } I_{90^{\theta}}^{1}\left(g_{c}\right) \geq 0 \\ 2, \quad I_{0}^{1}\left(g_{c}\right)<0 \text { and } I_{90^{\theta}}^{1}\left(g_{c}\right) \geq 0 \\ 3, \quad I_{{ }^{\theta}}^{1}\left(g_{c}\right)<0 \text { and } I_{90^{\theta}}^{1}\left(g_{c}\right)<0 \\ 4, I_{0}^{1}\left(g_{c}\right) \geq 0 \text { and } I_{90^{\theta}}^{1}\left(g_{c}\right)<0\end{array}\right.$

we have four possible directions depending on first order derivatives for each center pixel from above equation (8). The possible values can be $1,2,3$, or 4 , and finally, the image is converted into four values i.e., directions. The second-order $\mathrm{LTrP}\left(\mathrm{g}_{\mathrm{c}}\right)$ is defined as follow:

$\operatorname{LTrp}^{2}\left(g_{c}\right)=$
$\left\{f\left(I_{\text {dir }}^{1}\left(g_{c}\right), I_{\text {dir }}^{1}\left(g_{1}\right)\right), f\left(I_{\text {dir }}^{1}\left(g_{c}\right), I_{\text {dir }}^{1}\left(g_{2}\right)\right) \ldots \ldots . f\left(I_{\text {dir }}^{1}\left(g_{c}\right), I_{\text {dir }}^{1}\left(g_{p}\right)\right)\right.$

$\mathrm{f}_{3}\left(\mathrm{I}_{\text {dir }}^{\prime}\left(\mathrm{g}_{\mathrm{c}}\right), \mathrm{I}_{\text {dir }}^{\prime}\left(\mathrm{g}_{\mathrm{p}}\right)\right)=\left\{\begin{array}{l}0, \\ \mathrm{I}_{\text {dir }}^{\prime}\left(\mathrm{g}_{\mathrm{p}}\right),\end{array}\right.$

$$
\mathrm{I}_{\mathrm{dir}}^{\prime}\left(\mathrm{g}_{\mathrm{c}}\right)=\mathrm{I}_{\mathrm{dir}}^{\prime}\left(\mathrm{g}_{\mathrm{p}}\right)
$$

From (9) and (10), we get 8-bit tetra pattern for each center pixel, and then separate all patterns into four parts depending on the direction of center pixel. Finally, we get three binary patterns from tetra patterns.

Let the direction of center pixel obtained from equation (9) be "1"; then, LTrP2 can be defined by dividing it into three binary patterns as follows:

$$
\begin{gathered}
\left.\operatorname{LTrp}^{2}\right|_{\text {direction }}=2,3,4 \\
=\sum_{\mathrm{P}=1}^{8} 2^{\mathrm{P}-1} \times \mathrm{f}_{4}\left(\left.\operatorname{LTrp}^{2}\left(\mathrm{~g}_{\mathrm{c}}\right)\right|_{\text {direction }}\right)=234
\end{gathered}
$$

Same way other there tetra patterns for other directions of $g_{c}$ are get converted to binary patterns. Total we get 12 patterns $=4$ directions $\times 3$ patterns of each direction.

Finally similarity measurement by Euclidean Distance:

$$
\mathrm{D}=\sum_{\mathrm{t}=1}^{\mathrm{n}}|\mathrm{Xi}-\mathrm{Yi}|
$$

Estimation of magnitude component and histogram

The magnitude component \& sign component extracts more useful information, as it is proved that the combination can provide better results which are not evident in any one individual component. So we used the Thirteenth binary pattern which is calculated by using the magnitudes of horizontal and vertical first-order derivatives [17]

$$
M_{I} 1\left(g_{p}\right)=\sqrt{\left.\left(I_{0^{\theta}}^{1}\left(g_{p}\right)\right)^{2}+I_{90^{\theta}}^{1}\left(g_{p}\right)\right)^{2}}
$$

Local Pattern

$=\sum_{\mathrm{p}=1}^{\mathrm{p}} 2^{(\mathrm{p}-1)} \times \mathrm{f}\left(\mathrm{M}_{\mathrm{I}}^{1}\left(\mathrm{~g}_{\mathrm{p}}\right)-\mathrm{M}_{\mathrm{I}}^{1}\left(\mathrm{~g}_{\mathrm{c}}\right) \mid\right.$ direction $_{\mathrm{p}=8}$

For a pixel with p neighborhoods, possible combination of LBPs is $2^{(p)}$, which results the feature vector of length $2^{(p)}$. In order to reduce the computational cost uses the uniform patterns.

In this paper, those patterns which have equal to or less than two discontinuities in the circular binary representation is referred to as the uniform patterns, and the remaining patterns are referred to as non-uniform. Therefore, the uniform patterns for a given query image would be $\mathrm{p}(\mathrm{p}-1)+2$. The possible pattern which are uniform for $\mathrm{p}=8$ would be 58 .

At last stage after getting the local pattern, by constructing the histogram whole image is represented

$$
\begin{aligned}
& \frac{1}{\mathrm{~N}_{1} \times \mathrm{N}_{2}} \sum_{\mathrm{j}=1}^{\mathrm{N}_{1}} \sum_{\mathrm{k}=1}^{\mathrm{N}_{2}} \mathrm{f}(\operatorname{pattern}(\mathrm{j}, \mathrm{k}), \mathrm{l}) \\
& \mathrm{l} \in[0, \mathrm{p}(\mathrm{p}-1)+2]
\end{aligned}
$$

$$
\mathrm{N}_{1} \times \mathrm{N}_{2}=\text { size of input image }
$$

If both equal then 1or else o as shown in equation (10). We solve one image with the help of equation (7)-(15). As shown in figure 3.in which central pixel highlighted with red color where other horizontal and vertical with blue color.

We get three binary patterns as pattern 1,2 , and 3 shown in fig: 4.2. Referring to the generated $\mathrm{LTrP}$, the first pattern is obtained by keeping " 1 " where the tetra pattern value is " 2 " and " 0 " for other values, i.e., "00001000." In the same way, the other two )binary patterns "10100110" and "00010000" are computed for tetra pattern values " 3 " and " 4 ," respectively.

Similarly, tetra patterns for center pixels having directions 2, 3, and 4 are computed. Hence, we get four tetra patterns and 12 binary patterns. The magnitude of the first-order derivatives is used to get 13th binary pattern 


\section{Algorithm for Feature Extraction}

Algorithm:

1. Take the image and convert it into gray scale.

2. Apply first order derivatives in horizontal and vertical axis

3. Find the direction for every pixel i.e. 1, 2, 3 or 4

\begin{tabular}{|l|l|l|l|l|}
\hline 2 & 5 & 3 & 9 & 1 \\
\hline 6 & 7 & 7 & 2 & 5 \\
\hline 2 & 3 & 2 & 8 & 2 \\
\hline 3 & 8 & 3 & 2 & 9 \\
\hline 1 & 2 & 3 & 2 & 1 \\
\hline
\end{tabular}
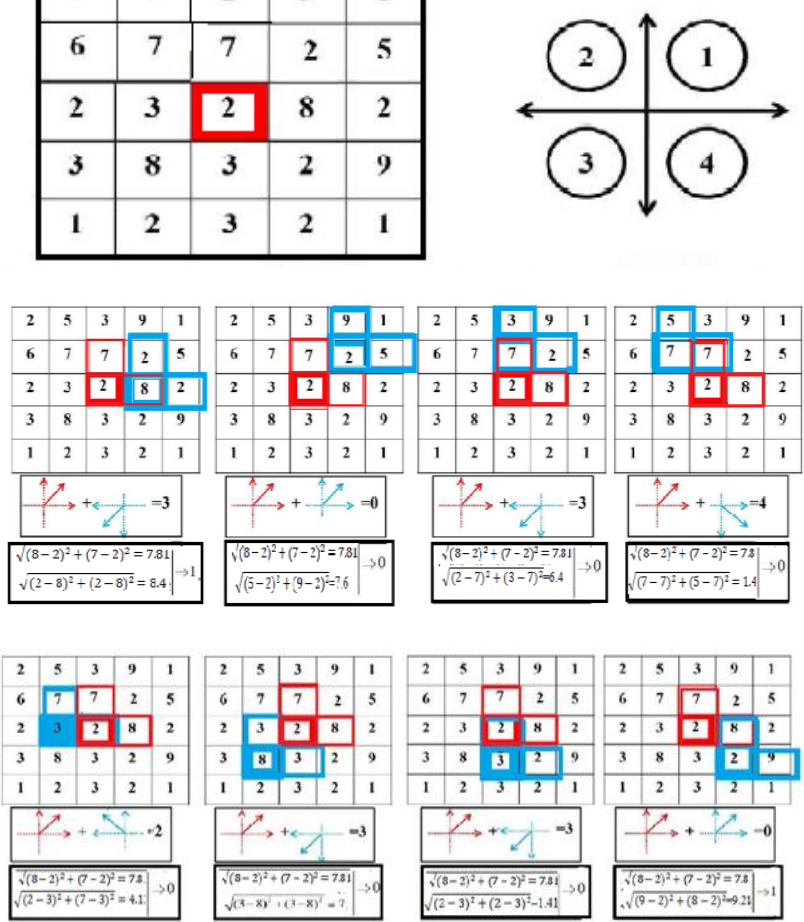

Tetra Pattern $=30342330$

Pattern1 = 00001000; Pattern2 = 10100110; Pattern $3=00010000$

Magnitude Pattern $=10000001$

Figure 3: Example to obtain the tetra and magnitude patterns

4. Depending on the direction of the center pixel the patterns get divided into four parts.

5. Calculate the tetra patterns, and separate them into three binary patterns.

6. Calculate the histograms of binary patterns.

7. Calculate the magnitudes of center pixels.

8. Construct the binary patterns, and plot their histogram

9. Take all histograms calculated from steps 6 and 8 .

10. Form the feature vector.

11. Query image Similarity matching with database image.

12. Based on the best matches retrieve the images from database.

Finally similarity measurement by Euclidean Distance: $\mathrm{D}=$ $\sum_{\mathrm{t}=1}^{\mathrm{n}}|\mathrm{Xi}-\mathrm{Yi}|$.To match top most similar matching query image with database Image by measuring distance between them.

\section{EXPERIMENTAL RESULTS AND DISCUSSION}

In the proposed work here, Matlab 7.6.0 is used for algorithm formation and evaluation. Other settings as on-board parallel processing unit RAM: 4GB, OS: Windows 7 and above. Collectively different types of 1000 Images are taken of kind coastal, forest, metro, desert for experimental result, to get similar content same as query mage taken by user when get compare with database image. We are dividing the result in two phase .Phase I concern with supervised content based image retrieval using defined pattern and Phase II concern with LTrP. Access outcome for query image as shown in Fig 4 query image taken, processed into gray scale and divide into 16 blocks then texture based retrieval, spectral based retrieval and finally with the help of both feature images get retrieved with more than $90 \%$ f-measure to have more effective result we applied LTrP on same data of 30 images among them we get 15 relevant images as shown in fig.(ix).

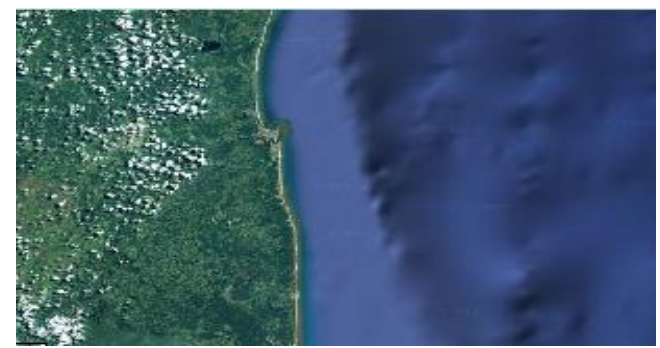

(i)

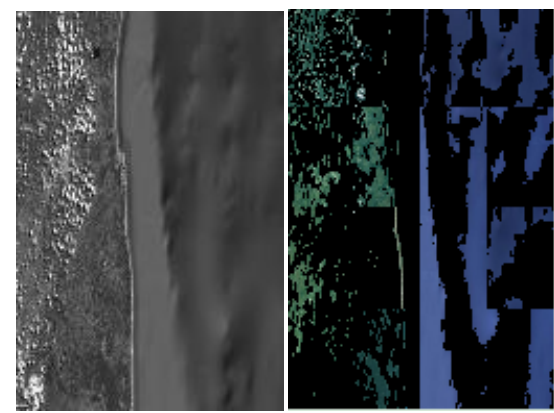

(ii)

(iii)

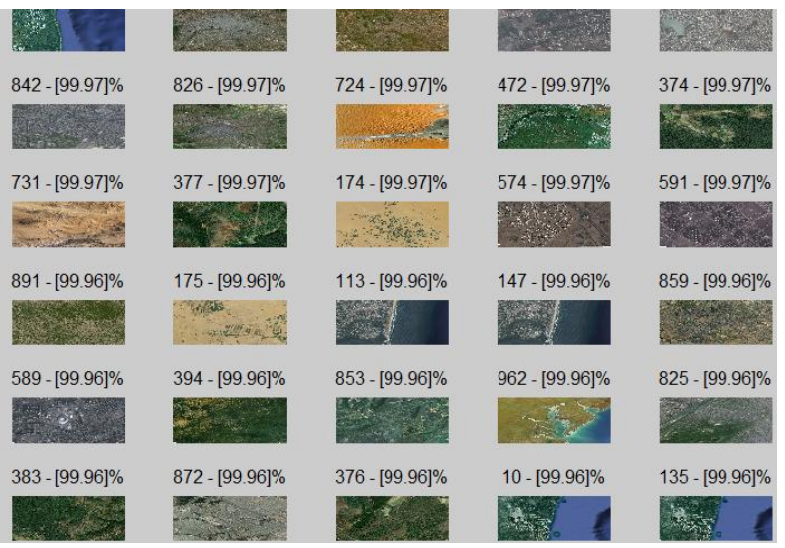

(iv) 


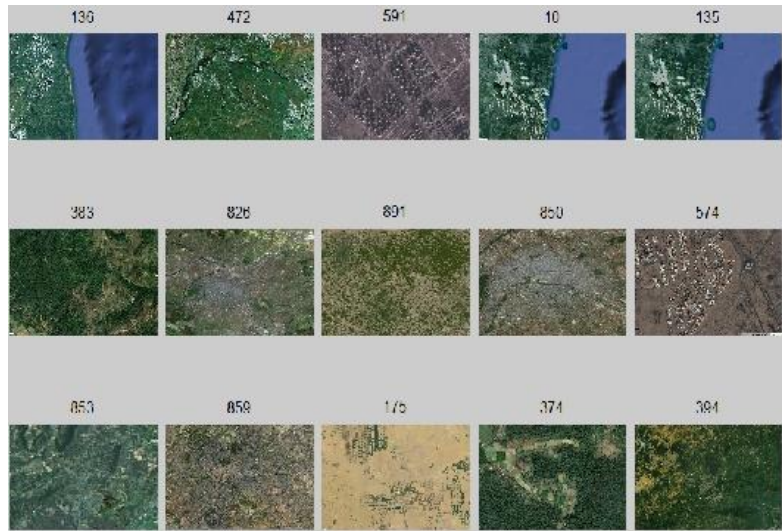

(v)

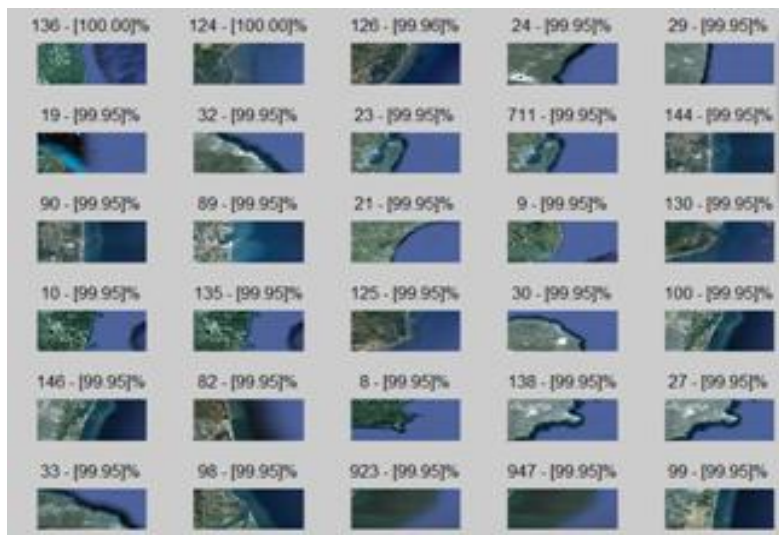

(vi)

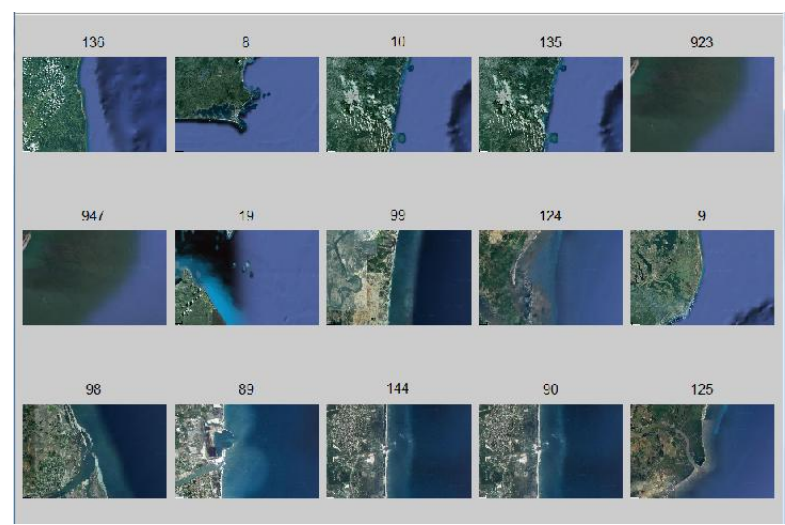

(vii)

\begin{tabular}{|c|c|c|c|c|}
\hline 136 - $\{100000$ & $144-100000 \%$ & $90-19996 \mathrm{k}$ & $10.19996 / 5$ & $135-19996 \%$ \\
\hline 2 & & & & As 35 \\
\hline 9.199 .9075 & $126-199.95 \mathrm{Ts}$ & $21.19995 \mathrm{~N}$ & $98-19995 \%$ & $124.9996 \%$ \\
\hline $32 \cdot .99 .95 \mathrm{~K}$ & 22. $19995 \mathrm{~K}$ & $125 \cdot 19995 / \%$ & $19-19995 \%$ & $89-199951 \%$ \\
\hline $24.19995 \mathrm{st}$ & $20.19995 \mathrm{~s}$ & $711-19995 \mathrm{rs}$ & $95.19995 \mathrm{~h}$ & $33.19995 \mathrm{~F}$ \\
\hline & & & & \\
\hline 130 - 199.9575 & $103.199 .95 \mathrm{k}$ & 8. $19995 \%$ & $28.19995 / \mathrm{k}$ & $145 \cdot$ - po $96 / 5$ \\
\hline Ex & & 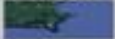 & & \\
\hline $96-19995 \%$ & $30-19995 \mathrm{~F}$ & $29.199 .96 \mathrm{~F}$ & $90 \cdot 199951 \%$ & $138-19996 \%$ \\
\hline & & & & \\
\hline
\end{tabular}

(viii)

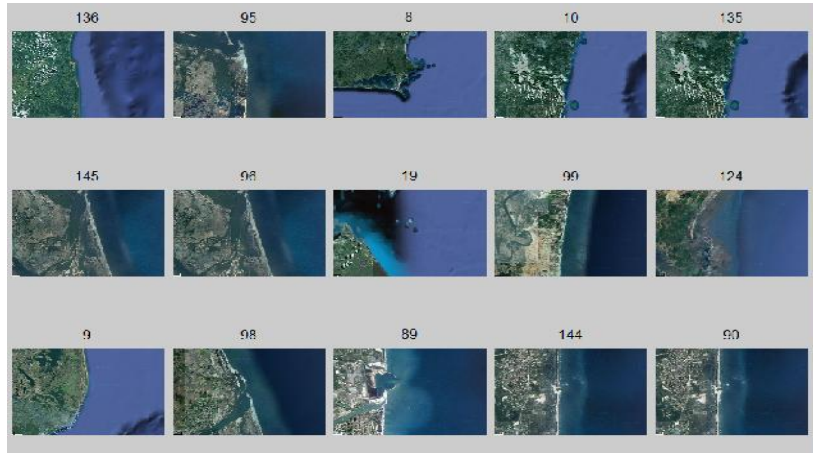

(ix)

Fig.4. Retrieved Outcome (i) Query Image (ii) Pre-processing of Query Image:-RGB Color Space to grey scale (iii) Query Image get divided in 16 blokes (iv) TF based extracted images after Phase I (v) TF based extracted images after Phase II (vi) SD based extracted images after Phase I (vii) SD based extracted images after Phase II (viii) TF \& SD based retrieved images after Phase I (ix) TF \& SD based retrieved images after Phase II

\subsection{Performance Analysis}

In this work, we have to consider the actual databases images to get 1000 remote control sensing images with every type has 250 images each. In this method 150 images are generally taken into consideration regarding instruction graphics and here 80 graphics are considered for screening images

Table I Proposed RSIR system performance retrieval results of costal area (ii) Desert (iii) Forest and (iv) Metro images

\begin{tabular}{|c|c|c|c|c|c|c|c|c|c|}
\hline \multirow{3}{*}{$\begin{array}{l}\text { Qu } \\
\text { ery } \\
\text { Im } \\
\text { age }\end{array}$} & \multicolumn{9}{|c|}{ Proposed RSIR Outcome Analysis } \\
\hline & \multicolumn{3}{|c|}{$\mathrm{TF}$} & \multicolumn{3}{|c|}{ SD } & \multicolumn{3}{|c|}{$\mathrm{TF} \& \mathrm{SD}$} \\
\hline & $\begin{array}{c}\text { Pre } \\
\text { cisi } \\
\text { on }\end{array}$ & $\begin{array}{c}\mathrm{Re} \\
\mathrm{cal} \\
1\end{array}$ & $\begin{array}{c}\mathrm{F}- \\
\mathrm{Me} \\
\text { asu } \\
\text { re }\end{array}$ & $\begin{array}{l}\text { Pre } \\
\text { cisi } \\
\text { on }\end{array}$ & $\begin{array}{c}\mathrm{Re} \\
\mathrm{cal} \\
1\end{array}$ & $\begin{array}{c}\text { F- } \\
\text { Me } \\
\text { asu } \\
\text { re }\end{array}$ & $\begin{array}{c}\text { Pre } \\
\text { cisi } \\
\text { on }\end{array}$ & $\begin{array}{c}\mathrm{Re} \\
\mathrm{cal} \\
1\end{array}$ & $\begin{array}{c}\mathrm{F}- \\
\mathrm{Me} \\
\text { asu } \\
\text { re }\end{array}$ \\
\hline $\begin{array}{l}\text { Co } \\
\text { ast } \\
\text { al } \\
\mathrm{Ar} \\
\text { ea }\end{array}$ & 50.1 & $\begin{array}{c}61 \\
.8 \\
4\end{array}$ & $\begin{array}{l}55 . \\
35\end{array}$ & 70.8 & $\begin{array}{c}88 \\
.9 \\
8\end{array}$ & $\begin{array}{l}78 . \\
85\end{array}$ & $\begin{array}{c}94.4 \\
2\end{array}$ & $\begin{array}{c}95 \\
.8\end{array}$ & $\begin{array}{c}95 . \\
1\end{array}$ \\
\hline $\begin{array}{c}\text { De } \\
\text { ser } \\
t\end{array}$ & 85.1 & $\begin{array}{r}95 \\
.9 \\
7\end{array}$ & $\begin{array}{l}90 . \\
23\end{array}$ & $\begin{array}{c}91.2 \\
8\end{array}$ & $\begin{array}{l}94 \\
.7 \\
7\end{array}$ & $\begin{array}{l}92 . \\
94\end{array}$ & 98.6 & $\begin{array}{l}89 \\
.8 \\
7\end{array}$ & $\begin{array}{l}94 . \\
06\end{array}$ \\
\hline $\begin{array}{l}\text { For } \\
\text { est }\end{array}$ & 89.8 & $\begin{array}{l}93 \\
.7 \\
8\end{array}$ & $\begin{array}{c}91 . \\
74\end{array}$ & $\begin{array}{c}95.4 \\
6\end{array}$ & $\begin{array}{l}98 \\
.3 \\
8\end{array}$ & $\begin{array}{l}96 . \\
89\end{array}$ & $\begin{array}{c}99.8 \\
9\end{array}$ & $\begin{array}{l}98 \\
.7 \\
9\end{array}$ & $\begin{array}{l}99 . \\
33\end{array}$ \\
\hline $\begin{array}{l}\mathrm{Me} \\
\text { tro }\end{array}$ & $\begin{array}{c}81.7 \\
8\end{array}$ & $\begin{array}{l}96 \\
.3 \\
9\end{array}$ & $\begin{array}{c}88 . \\
48\end{array}$ & $\begin{array}{c}79.9 \\
7\end{array}$ & $\begin{array}{l}97 \\
.9 \\
3\end{array}$ & $\begin{array}{l}88 . \\
04\end{array}$ & $\begin{array}{c}91.6 \\
9\end{array}$ & $\begin{array}{l}98 \\
.8 \\
9\end{array}$ & $\begin{array}{r}95 . \\
15\end{array}$ \\
\hline
\end{tabular}

As shown in above Table.1 this three parameters [18] shows the retrieval rate which can be visualize by following Figure. Figure 5 Shows parameter analysis of Texture Feature gives more than $90 \%$ retrieval result for desert, forest and metro. Figure 6 shows parameter analysis of spectral distribution gives more than $90 \%$ retrieval result coastal, desert, forest. Figure 7 shows parameter analysis of both texture and spectral feature gives $90 \%$ and above result for all type of images i.e. coastal, desert, forest, metro. 


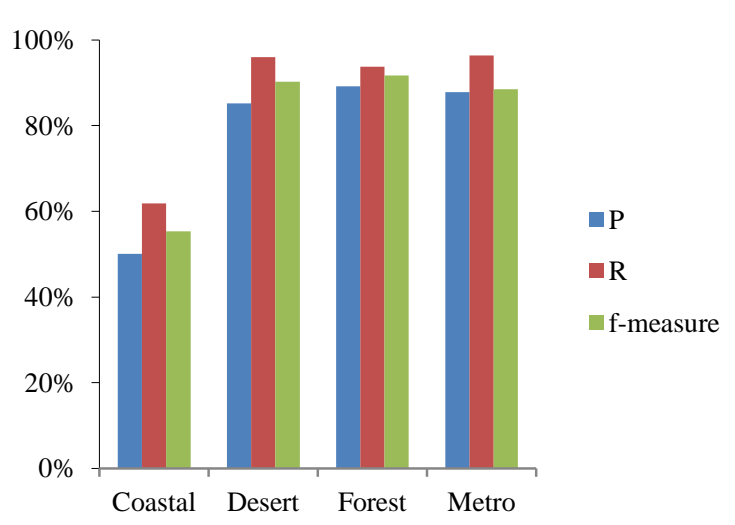

Figure 5: TF

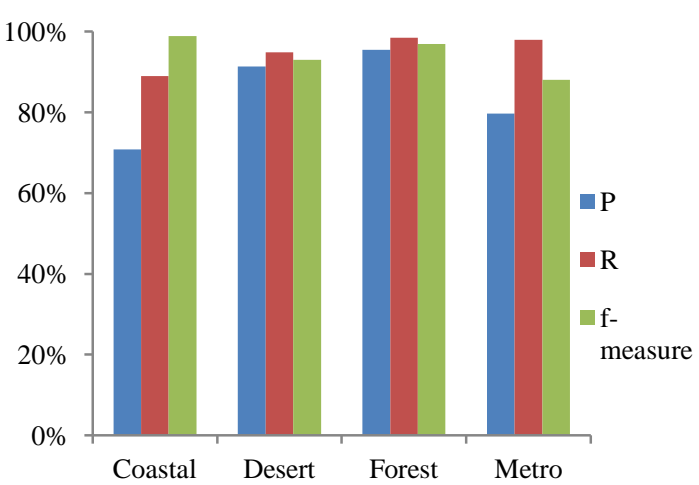

Figure 6. SD

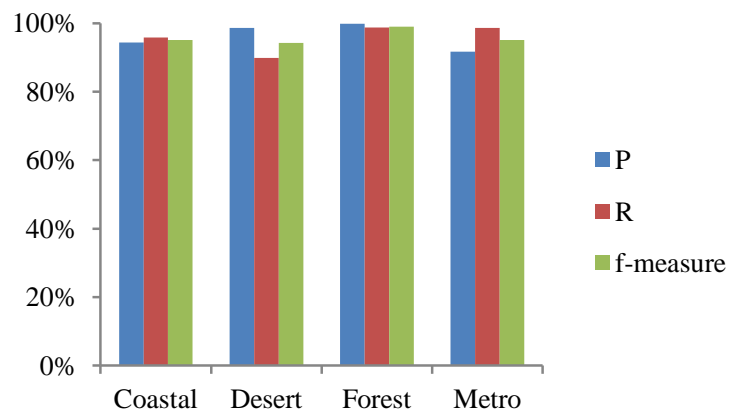

Figure7: TF \& SD

Now we will see comparative study of two phases with the help of f-measure parameter. As shown in fig 5.5 to fig.5.7comparison of f-measure which we get form phase-I is done with f-measure of retrieval result after applying LTrP which is conducted in phase-II for each feature TF, SD, TF and $\mathrm{SD}$ respectively.

Table 2 Proposed RSIR System Performance Retrieval Results After second Filtration of Costal Area (ii) Desert (iii) Forest and (iv) Metro images

\begin{tabular}{|c|c|c|c|c|c|c|c|c|c|}
\hline \multirow{2}{*}{$\begin{array}{c}\text { Que } \\
\text { ry } \\
\text { Ima } \\
\text { ge }\end{array}$} & \multicolumn{3}{|c|}{ TF } & \multicolumn{3}{|c|}{ Troposed RSIR Outcome Analysis for Phase II } \\
\cline { 2 - 8 } & $\begin{array}{c}\text { Preci } \\
\text { sion }\end{array}$ & $\begin{array}{c}\text { Rec } \\
\text { all }\end{array}$ & $\begin{array}{c}\text { M- } \\
\text { sea } \\
\text { sure }\end{array}$ & $\begin{array}{c}\text { Preci } \\
\text { sion }\end{array}$ & $\begin{array}{c}\text { Rec } \\
\text { all }\end{array}$ & $\begin{array}{c}\text { M- } \\
\text { Mea } \\
\text { sure }\end{array}$ & $\begin{array}{c}\text { Preci } \\
\text { sion }\end{array}$ & $\begin{array}{c}\text { Rec } \\
\text { all }\end{array}$ & $\begin{array}{c}\text { F- } \\
\text { Mea } \\
\text { sure }\end{array}$ \\
\hline
\end{tabular}

\begin{tabular}{|c|c|c|c|c|c|c|c|c|c|}
\hline $\begin{array}{c}\text { Coa } \\
\text { stal } \\
\text { Are } \\
\text { a }\end{array}$ & 52.1 & $\begin{array}{c}69 . \\
84\end{array}$ & $\begin{array}{c}59.6 \\
8\end{array}$ & 74.8 & $\begin{array}{c}89 . \\
98\end{array}$ & $\begin{array}{c}81.6 \\
9\end{array}$ & 96.4 & $\begin{array}{c}95 . \\
8\end{array}$ & $\begin{array}{c}96.1 \\
0\end{array}$ \\
\hline $\begin{array}{c}\text { Des } \\
\text { ert }\end{array}$ & 88.1 & $\begin{array}{c}98 . \\
97\end{array}$ & $\begin{array}{c}93.2 \\
2\end{array}$ & 93.28 & $\begin{array}{c}96 . \\
78\end{array}$ & $\begin{array}{c}95.0 \\
0\end{array}$ & 99.5 & $\begin{array}{c}90 . \\
87\end{array}$ & $\begin{array}{c}94.9 \\
9\end{array}$ \\
\hline $\begin{array}{c}\text { Fore } \\
\text { st }\end{array}$ & 90.8 & $\begin{array}{c}99 . \\
78\end{array}$ & $\begin{array}{c}95.0 \\
8\end{array}$ & 94.46 & $\begin{array}{c}99 . \\
8\end{array}$ & $\begin{array}{c}97.0 \\
6\end{array}$ & 98.98 & $\begin{array}{c}99 . \\
79\end{array}$ & $\begin{array}{c}99.3 \\
8\end{array}$ \\
\hline $\begin{array}{c}\text { Met } \\
\text { ro }\end{array}$ & 83.78 & $\begin{array}{c}99 . \\
39\end{array}$ & $\begin{array}{c}90.9 \\
2\end{array}$ & 80.97 & $\begin{array}{c}97 . \\
99\end{array}$ & $\begin{array}{c}88.6 \\
7\end{array}$ & 96.68 & $\begin{array}{c}97 . \\
99\end{array}$ & $\begin{array}{c}97.3 \\
3\end{array}$ \\
\hline
\end{tabular}

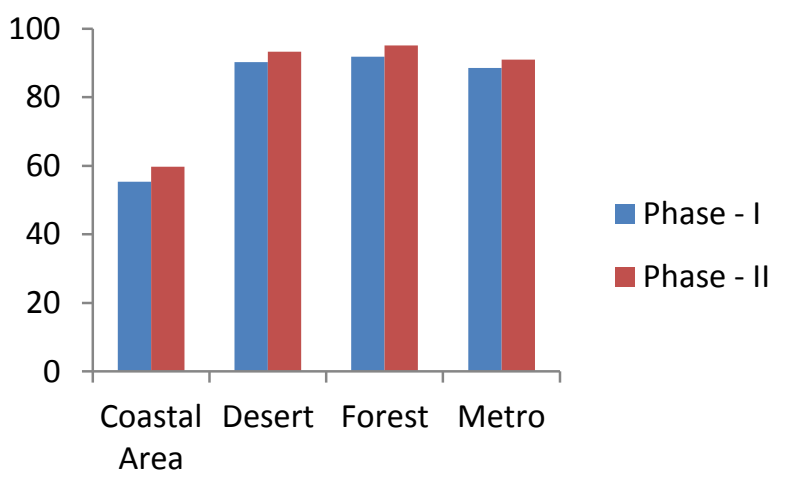

Figure 8: f measure-TF Table 5.2 and Table 5.3

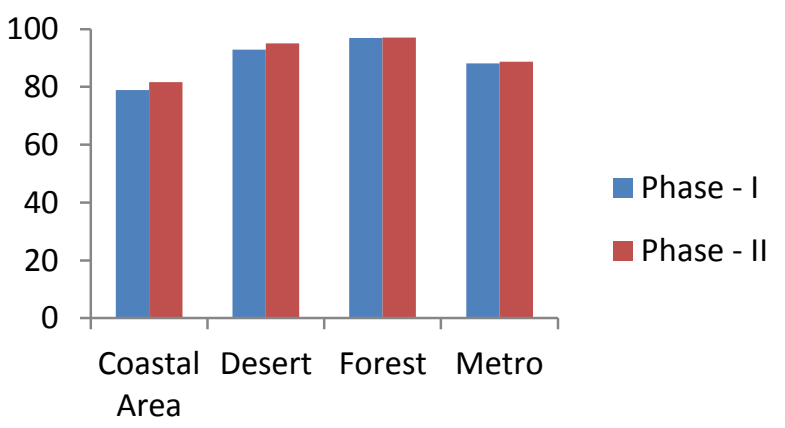

Figure 9: f measure - SD Table 5.2 and Table 5.3

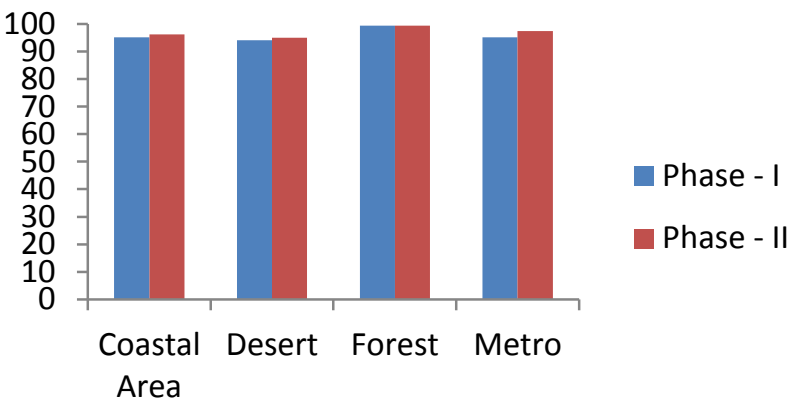

Figure10: $f$ measure - TF and SD Table 5.2 and Table 5.3

\section{DISCUSSION}

Each of our proposed technique overall performance effects based on three features TF, SD and combination are mentioned in Table 1. In overall performance analysis metrics each of our proposed new patterns based RSIR system offers high detail along with recall rate in comparison with individual features. As shown in Fig 5 to Fig 7 combine result of TF and SD shows best 
result shown in Fig 7.

This result is after Phase I, to have proper ordering of retrieve images Phase II get applied on result of Phase I, as shown in fig: 8 to Fig 10. Fig.10 shows result after Phase- II, with better ordering as compare to Phase- I retrieval image rendering ordering which is based on LTrP techniques

\section{CONCLUSION}

We proposed a system with two Phase implementation,

Phase I: Supervised Retrieval using piecewise defined pattern similarities

Feature Extraction:

Spectral Distribution (SD)-Using ASMC

Texture Feature (TF)-Using Gabor Function

Texture extraction by Two-Dimensional Gabor function which used generally .Spectral Distribution uses Advanced parallelizes split and merge clustering Algorithm, due to which we get fast response time and avoid single point fault. Parallelization achieve through Master and Slave architecture. As shown in Fig 7 overall combine result of both TF and SP gives very well relevant result as shown in Table $1 \mathrm{f}$-measure give count above $90 \%$ as compare to individual feature's result. Result also depends on type of data i.e. type of image. Phase II: Relevance feedback through LTrP

When LTrP get applied over result of Phase I i.e. most relevant images as query image from trained database, we get most relevant images of that retrieved images in Phase I. This is based on $\mathrm{LTrP}$

\section{REFERENCES}

[1] T. Bretschneider, R. Cavet, O. Kao, "Retrieval of remotely sensed imagery using spectral information content", Proceedings of the InternationalGeosciences and Remote Sensing Symposium, vol. 4, pp. 2253-2256, 2002.

[2] B. Liu, T. Bretschneider, "D-ISMC: A distributed unsupervised classification algorithm for optical satellite imagery", Proceedings of theInternational Geosciences and Remote Sensing Symposium 2003, in press

[3] Y. Rubner, C. Tomasi, L.J. Guibas, "The Earth Mover's Distance as a metric for image retrieval", International Journal of Computer Vision, vol. 40, no. 2, pp. 99-121, 2000.

[4] O. Kao, T. Bretschneider, G.R. Joubert, "Image retrieval with Gabor- Wavelet- Networks", Proceedings of the International Conference on Internet and Multimedia Systems and Applications, pp. 312-317, 2002.

[5] T.S. Lee, "Image representation using 2D Gabor wavelets", IEEE Transactions on Pattern Analysis and Machine Intelligence, vol. 18, no. 10, 959-971, 1996.

[6] Y. Rui, T. S. Huang, S. Mehrotra, "Content-based image retrieval with relevance feedback in MARS", IEEE Transaction on Image Processing, vol. 9, pp. 102- 119, 2000.

[7] P. Muneesawang, L. Guan, "Interactive CBIR using RBFbased relevance feedback for WT/VQ coded images", Proceedings of the IEEE International Conference on Acoustic, Speech and Signal Processing,vol. 3, pp. 16411644, 2001.

[8] H. Greenspan, G. Dvir, Y. Rubner, "Region correspondence for image matching via EMD f low", Proceedings of IEEE Workshop on Contented- based Access of Image and Video Libraries, pp. 27-31, 2000.

[9] "SRBIR", Journal of Computer Science, Felci Rajam et al., 2011a.

[10] Z. Guo, L. Zhang, and D. Zhang, "A completed modeling of local binary pattern operator for texture classification," IEEE Trans. Image Process., Vol. 19, No. 6, pp. 16571663, June 2010.

[11] "Scale and Rotation Invariant Gabor Features for Texture Retrieval", IEEE Conference DICTA, Rahman M.H. et al., 2011

[12] "Memory Learning Framework for Retrieval of Neural Objects" International Journal of Advanced Research in Computer Engineering \& Technology (IJARCET), Sanjeev S. Sannakki, Sanjeev P. Kaulgud, Volume 1, Issue 6, August 2012.

[13] Junwei Han, King N. Ngan, Fellow, IEEE, Mingjing Li, and Hong-Jiang Zhang, Fellow, IEEE, A Memory Learning Framework for Effective Image Retrieval. 2007.

[14] En cherg, Feng Jing, Chao, Lei Zhang, "search Result clustering based relevance feedback for web image retrieval",IEEE, 1-422-0728-1/07/2007

[15] H. J. Zeng, Q. C. He, Z. Chen, W. Y. Ma and J. W. Ma, "Learning to cluster Web search results," Proc. of the $27 \mathrm{~h}$ annual international ACM SIGIR conference, pp. 210-217.

[16] Srikant S.K ,Dr T C Manjunath, “A Novel improved technique of image indexing for efficient content based image retrieval using local patterns", IJETI-Volume 12 Number 9- Jun 2014

[17] Subramanian Murala, R.P.Maheshwari and R.Balasubramanian, "Local Tetra Patterns: A New Feature Descriptor for Content-Based Image Retrieval",IEEE Trans. Image Process., vol. 21, no. 5, pp. 2874-2886, MAY 2012.

[18] http://en.wikipedia.org/wiki/Information_retrieval.

[19] http://en.wikipedia.org/wiki/Contentbased_image_retrieval. 